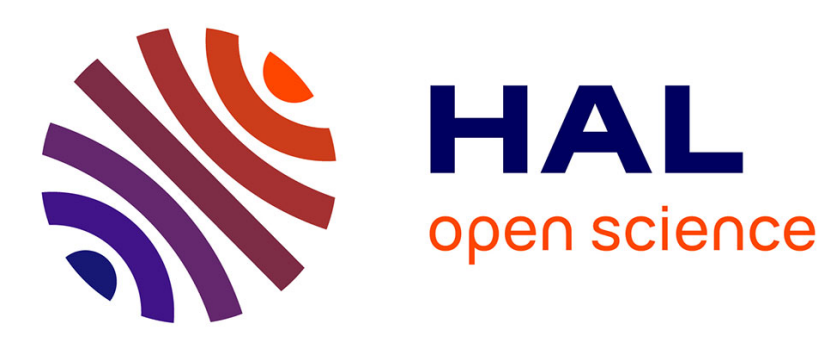

\title{
Non-linear electron spin resonance study of archaebacteria lipid dynamics
}

\author{
S. Bruno, A. Gliozzi, S. Cannistraro
}

\section{To cite this version:}

S. Bruno, A. Gliozzi, S. Cannistraro. Non-linear electron spin resonance study of archaebacteria lipid dynamics. Journal de Physique, 1986, 47 (9), pp.1555-1563. 10.1051/jphys:019860047090155500 . jpa-00210355

\section{HAL Id: jpa-00210355 https://hal.science/jpa-00210355}

Submitted on 1 Jan 1986

HAL is a multi-disciplinary open access archive for the deposit and dissemination of scientific research documents, whether they are published or not. The documents may come from teaching and research institutions in France or abroad, or from public or private research centers.
L'archive ouverte pluridisciplinaire HAL, est destinée au dépôt et à la diffusion de documents scientifiques de niveau recherche, publiés ou non, émanant des établissements d'enseignement et de recherche français ou étrangers, des laboratoires publics ou privés. 


\title{
Non-linear electron spin resonance study of archaebacteria lipid dynamics
}

\author{
S. Bruno $\left({ }^{+}\right)$, A. Gliozzi $\left({ }^{+}\right)$and S. Cannistraro $\left({ }^{++}\right)$ \\ $\left({ }^{+}\right)$Dipartimento di Fisica dell'Università, Genova, Italy \\ $\left({ }^{++}\right)$Dipartimento di Fisica dell'Università, Perugia, Italy
}

(Reçu le 11 mars 1986, accepté le 23 mai 1986)

\begin{abstract}
Résumé. - On utilise la diffusion spectrale qui apparaît dans les expériences de résonance paramagnétique électronique lorsque le champ magnétique est modulé à une fréquence adéquate, afin d'obtenir des informations sur la dynamique d'une classe inhabituelle de lipides qui sont extraits de bactéries vivant dans des conditions physiques extrêmes (haute température et très bas $\mathrm{pH}$ ). Ces lipides sont très différents des lipides usuels qui sont proches des acides gras, et leur structure moléculaire semble jouer un rôle remarquable pour la stabilité de la membrane plasmique de ces bactéries. Une détermination complète des paramètres expérimentaux permet de calculer le temps de corrélation rotationnel de lipides marqués par un spin électronique qui limitent le comportement dynamiquè des lipides de ces bactéries. On trouve que les lipides étudiés ont des mouvements moléculaires anisotropes caractérisés par un temps de corrélation très long même à des températures très élevées. Les mouvements anisotropes très lents de ces liquides indiquent que leurs têtes polaires nonitol sont capables d'établir entre elles des interactions fortement coopératives; d'un point de vue biophysique, ces interactions pourraient être invoquées pour expliquer la grande stabilité de la membrane plasmique dans ces conditions extrêmes.
\end{abstract}

\begin{abstract}
The spectral diffusion occurring in non-linear electron spin resonance experiments performed at a suitable frequency of field modulation is exploited to gain dynamical information on an unusual class of lipids extracted from bacteria living in extreme physical conditions (high temperature and very low $\mathrm{pH}$ ). These lipids are quite different from the usual fatty acid lipids and their molecular structure seems to play a particular role in the stability of the plasma membrane of these bacteria. An accurate and extensive determination of the magnetic instrumental parameters allowed a reliable calculation of the rotational correlation time of suitable spin labelled lipids which mimic the dynamical behaviour of the lipids under investigation. The lipids investigated have been shown to undergo an anisotropic molecular motion characterized by a very slow correlation time even at very high temperatures. The very slow anisotropic motion of these lipids indicates the ability of their nonitol polar heads to establish strong cooperative interactions which, from a biophysical point of view, could be invoked to explain the great stability of the plasma membrane even under those extreme environmental conditions.
\end{abstract}

\section{Introduction.}

The characterization of the dynamic properties of constituent biomolecules plays a major role in elucidating the structure and function of biological systems. A great deal of information about molecular dynamics, especially in lipids, membranes and proteins has been acquired through the application of the Electron Spin Resonance (ESR) spin labelling technique. This method consists in placing a nitroxide group, bearing a stable free electron, in the system of interest either by attaching it covalently to a molecule (spin label) or by introducing into the system a suitably tailored radical without permanent bonding to any molecule (spin probe). The location of the spin label or spin probe in the host system can generally be well defined and the analysis of ESR line positions and line shapes, either in isotropic or anisotropic systems, can yield information about the rate of molecular motion, structure, order, viscosity and polarity of the host system.

As compared with other techniques, spin labelling has great advantages because of its ability to describe motions over a broad range of time scales, i.e. for rotational correlation times between $10^{-11}$ and $10^{-4} \mathrm{~s}$. For convenience this range has been subdivided into three different parts : values of $\tau_{\mathrm{c}} \approx 3 \times 10^{-9} \mathrm{~s}$ define the fast motional region $3 \times 10^{-9} \approx \tau_{\mathrm{c}} \approx 10^{-7} \mathrm{~s}$ the slow motional region and $\tau_{\mathrm{c}}$ values longer than $10^{-7} \div 10^{-6} \mathrm{~s}$ the very slow region. 
The rate of molecular tumbling determines the positions and the widths of the nitroxide ESR resonance lines, and for spin labels in isotropic media (i.e. liquids) the theory has been well developed [1]. In lipid systems and biological membranes, where a molecular ordering exists, the motion can be anisotropic. As a result, significant changes in the spectroscopic properties of nitroxide spin labels can occur, as compared with the spectra obtained in isotropic systems, thereby enhancing the complexity of the theory.

When the spin label motion is fast enough $\left(\tau_{\mathrm{c}} \approx 3 \times\right.$ $\left.10^{-9} \mathrm{~s}\right)$ the linewidth can be accurately described by treating the time-dependent spin rotational term of the spin Hamiltonian as a perturbation to the Zeeman and hyperfine levels (Redfield approach [2]) and by then solving the equation of the evolution of the spin density matrix [3-5]. These calculations require the assumption of a particular model for the molecular reorientation and, in anisotropic systems, of the orienting potential [6-8].

For slower molecular motions $\left(3 \times 10^{-9} \mathrm{~s} ₹ \tau_{\mathrm{c}} \approx\right.$ $\left.10^{-7} \mathrm{~s}\right)$ the time-dependent part of the spin Hamiltonian does not fluctuate rapidly enough and the perturbation theory of Redfield is no longer valid. The simplest approach for a theoretical analysis of the ESR response is to solve the Bloch equations, modified by adding a diffusion term [9]; however, this theory does not take into account pseudo-secular transitions [1]. A more powerful treatment is based on the stochastic Liouville equation that describes the equation of the motion of the spin density matrix [10] and has been solved for isotropic media by using approximate numerical methods [11-13]. In the case of anisotropic systems the theory becomes more complex [10, 14-15] and extensive simulations are involved, requiring large amounts of computer time and storage [16-17]. Approximate methods for evaluating $\tau_{\mathrm{c}}$ without spectra simulation have been proposed, both for isotropic and for simpler cases of anisotropic systems, provided that some assumptions are made about the molecular reorientation [9-10, 17-18]. Conventional ESR spectroscopy is sensitive to molecular motions with rates greater than $10^{7} \mathrm{~s}^{-1}$. However, in order to obtain detailed insight into many important biological processes involving rotational correlation times of microseconds or longer, techniques sensitive to slower motions are needed. In this respect nonlinear ESR spectroscopies have been developed, obtaining spectral lineshapes sensitive to $\tau_{\mathrm{c}}$ values in the very slow motion range $\left(10^{-7} \approx \tau_{c} ₹ 10^{-3} \mathrm{~s}\right)$. This branch of ESR spectroscopy is referred to as Saturation Transfer ESR (ST-ESR) because the signal response is strongly affected by the diffusion of saturation between different portions of the resonance spectrum. One method for measuring saturation transfer is continuous wave saturation [19], in which the ordinary ESR absorption is observed as a function of microwave field intensity; but this method is not very sensitive to motions slower than $10^{-6} \mathrm{~s}$. A more direct method is to perform a continuous wave electron-electron double resonance (ELDOR) [20] experiment in which one portion of the spectrum is saturated by the application of an intense microwave field, and the effects of this saturation at other points in the spectrum are investigated using a second microwave bridge. However, the ELDOR method is instrumentally complex and the geometry of the bimodal cavities necessary for this kind of experiment has not been optimized for aqueous samples.

Finally, starting in 1971, there has been developed the so-called adiabatic rapid passage technique by which the effects of saturation transfer on the response of a spin system that is undergoing passage [21] through the resonance condition due to the application of a sinusoidal modulation of the Zeeman magnetic field can be studied. This passage method is based on the competition between field modulation and rotational diffusion in governing the passage of spins through the resonance condition. Phase-sensitive detection is used to select the portion of the signal that arises only from saturation effects and is therefore selectively to saturation transfer rates that are about in the same range as the modulation frequency. Thomas et al. [22] showed that the second harmonic absorption out-of-phase display represents the most suitable and sensitive display in saturation transfer spectroscopy and introduced a commercial spectrometer which allows this kind of experiment to be performed with nearly the same ease as conventional ESR ones.

ST-ESR spectra can be calculated by taking into explicit account the effects of field modulation and saturation in the theory. Again the simplest approach is to start with the diffusion-coupled Bloch equations, except that now the constant magnetic field is replaced by a sinusoidal one [23]. A more advanced simulation method uses the stochastic Liouville theory [24-25] that has been extended to anisotropic rotational diffusion [26]. Current simulation of ST-ESR spectra of nitroxide spin labels requires a great demand for computer time and memory [27-28], but the development of fast computer programs, based on suitable approximations in the theory, is in progress. Some advances have been published recently [29-30]. Thomas et al. [22] have introduced a method for obtaining very slow rotational correlation times from the ratio of the spectral parameters $C^{\prime} / C, L^{\prime \prime} / L$ and $H^{\prime \prime} / H$ (shown in Fig. 4) measured on second harmonic, $90^{\circ}$ out-of-phase, absorption spectra. In fact, they have constructed calibration curves of these line shape parameters against $\tau_{\mathrm{c}}$, deriving these latter from experimental reference spectra of spin-labelled hemoglobin in solvents of an increasing viscosity [22]. For isotropically reorienting labels the three independent values of $\tau_{c}$ obtained from the three calibration curves are equal within the limits of experimental error. If the molecular motion in the slow rate domain shows 
a behaviour of an anisotropic nature, the problems encountered are still far from being solved. In this case, in fact, different effective correlation times are obtained from calibration curves corresponding to the different ratios [22]. A method followed in the literature consists in performing an analysis of anisotropic motion by using the isotropic reference data $[25,31]$; however this procedure can sometimes be misleading in the estimation of correlation times [32]. However, the most straightforwardly computable ratio $C^{\prime} / C$ arising from a stearic acid spin label undergoing anisotropic motion in a lipid dispersion has been shown to exhibit a behaviour qualitatively similar to that of the isotropic motion model [33]. The use of Thomas et al. [22] calibration curves requires an accurate spectrometer setting, in order to work in their same instrumental conditions. Indeed, since the line shape of ST-ESR spectra is critically dependent upon the extent and distribution of the microwave magnetic field and the modulation amplitude [34-36], an exact calibration of these parameters has proved to be necessary.

We have performed an ST-ESR spin label study of bipolar lipids extracted from Sulfolobus solfataricus, an extreme thermo-acidophilic archaebacterium growing at about $85^{\circ} \mathrm{C}$ and $\mathrm{pH} 3$, in order to detect the presence of molecular rotational diffusion in the very slow motion region. The chemical structure of these lipids is remarkable different from that of normal lipids [37] and is shown in figure 1. This confers on the lipids very peculiar features from the dynamical and structural points of view [37-39]. By means of the different methods discussed in the present paper, we carried out accurate measurements of the magnetic instrumental parameters upon which the ST-ESR spectra are critically dependent $[22,34-36,40]$; we then recorded the conventional ESR and ST-ESR spectra of our spin-labelled lipid system as a function of temperature. We found that GDNT lipids, arranged in random dispersions, undergo very slow anisotropic molecular motions and, surprisingly enough, this rate, as compared with ordinary lipids, remains low even at what from a biological point of view are very high temperatures. The biophysical importance of this behaviour, which appears unique up to now in the literature, is discussed.

\section{Experimental methods.}

Glycerol-dialkyl-nonitol-tetraether (GDNT) was extracted from Sulfolobus solfataricus and purified according to procedures previously described [37]. It consists of two $\mathrm{C}_{40 \omega-\omega}$, biphytanyl residues having two polar heads (one glycerol and one nonitol). The hydrophobic chain may contain from zero up to four cyclopentane rings per chain. The 5-doxylstearic acid spin label (5SASL) was purchased from SYVA Res. Chem. (Palo Alto) (its formula is shown in Fig. 1) and 1,1-diphenyl-2-picrylhydrazil (DPPH) from Fluka (Switzerland). N-methylphenazinium tetracyano-qui-

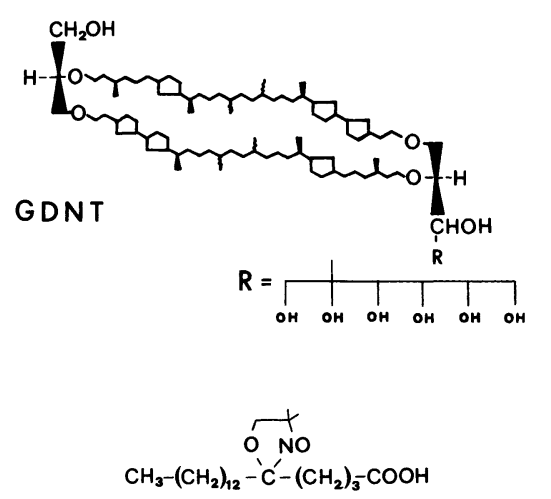

5SASL

Fig. 1. - Chemical formulae of the lipid investigated, GDNT, and of the spin-labelled lipid used as probe, 5SASL. The hydrophobic chain of the GDNT lipids may contain from zero up to four cyclopentane rings per chain [37].

nodimethane crystals (NMP-TCNQ) were a gift from Dr. A. I. Vistnes (Oslo). Peroxylamine disulphonate (PADS) was from Aldrich Chemical Co. (Belgium). A solution of GDNT was prepared by dissolving it in chloroform at a concentration of $10 \mathrm{mg} / \mathrm{ml}$, while the spin labels were dissolved in ethanol. PADS was dissolved at a concentration of $1 \mathrm{mM}$ in nitrogensatured water containing $10 \mathrm{mM} \mathrm{K}_{2} \mathrm{CO}_{3}$.

To prepare ESR samples, aliquots of label solution were added to lipid solution to reach different label/ lipid molar ratios. The optimal label/lipid molar ratio was chosen in order to minimize dipolar interactions and maximize signal amplitude; it was found to be about $1 \%$. The dependence of second harmonic, $90^{\circ}$ out-of-phase, absorption Saturation Transfer ESR spectra on sample size and position arising from inhomogeneities in the microwave and modulation field in the cavity [35-36] made it necessary to work with " point samples " positioned exactly in the centre of the cavity. 2-mm long samples in a microcapillary were therefore prepared by successively evaporating small amounts of the lipid-label mixture so as, in the end, to use a total quantity of about $10 \mu 1$. The sample, consisting in dry lipid molecules containing 5 SASL spin probe, was introduced into a standard $4 \mathrm{~mm}$ ESR quartz tube which was, in turn, put in the temperature control quartz dewar. In this way the sample was placed in the centre of the resonant $\mathrm{TE}_{102}$ cavity of the spectrometer. The sample temperature was regulated by a nitrogen flux and monitored by a platinum thermo-resistor placed immediately below the ESR tube. The temperature gradient across the sample was found not to exceed $0.5 \circ \mathrm{C} / \mathrm{cm}$, the sample being always placed at the same position in the cavity. Thermodynamic equilibrium was reached by equilibrating the sample for $6 \mathrm{~min}$ after each change in temperature. In this way no hysteresis effects, depending on direction of temperature variation, were detected. 
ESR spectra were recorded by an X-band Varian E-109 spectrometer. A $100 \mathrm{kHz}$ modulation frequency was used for conventional first harmonic, in-phase, absorption ESR spectra. The spectrometer used was moreover equipped with $50 \mathrm{kHz}$ field modulation and phase control facilities which are necessary for performing ST-ESR experiments. Such experiments, in the second harmonic, $90^{\circ}$ out-of-phase, absorption mode were conducted at a modulation frequency of $50 \mathrm{kHz}$ and receiver frequency of $100 \mathrm{kHz}$, at a modulation amplitude of $5 \mathrm{G}$ and at a microwave magnetic field strength of $0.25 \mathrm{G}$.

A detailed calibration of the experimental settings was needed. First, we wanted an exact determination of the microwave magnetic field, $H_{1}$, incident on the sample. Theoretically, the average value of the microwave magnetic field $\left\langle H_{1}^{2}\right\rangle_{\mathrm{s}}$ at the sample may be related to the average value of $\left\langle H_{1}^{2}\right\rangle_{\mathrm{w}}$, the microwave magnetic field propagating along the waveguide, which is, in turn, proportional to the microwave power $P$. Therefore the following relationship holds [41]

$$
\left\langle H_{1}^{2}\right\rangle_{\mathrm{s}}=2 \times 10^{-3} P Q_{\mathrm{L}} \eta\left(V_{\mathrm{w}} / V_{\mathrm{s}}\right)\left(1-|\Gamma|^{2}\right) .
$$

In equation (1), $\eta$ is the filling factor, $V_{\mathrm{s}}$ and $V_{\mathrm{w}}$ are the sample volume and the volume of a section of waveguide on guide wavelength long respectively, $Q_{\mathrm{L}}$ is the loaded cavity $Q$ factor and $\Gamma$ the reflection coefficient at the cavity. Since the determination of these parameters is very difficult, a direct experimental measurement of the microwave magnetic field incident on the sample is required. We followed the method of perturbing spheres [42] which allows a determination of the microwave magnetic field strength at a position in the cavity by monitoring the resonant cavity frequency shift induced by a small metallic sphere placed at that position, according to the equation [42]

$\left\langle H_{1}^{2}\right\rangle_{\mathrm{s}}=\left[\left(v^{2}-v_{0}^{2}\right) / v_{0}^{2}\right]\left\{\mu P_{\mathrm{i}} / 4 \pi^{2}\left(v_{2}-v_{1}\right) a^{3}\right\} \times 10^{8}$

where all the parameters are in MKSA units with the exception of $H_{1}$ which is expressed in gauss. In equation (2), $v_{0}$ is the unperturbed cavity resonance frequency, $v$ the perturbed cavity resonance frequency, $a$ the radius of the perturbing sphere, $P_{\mathrm{i}}$ the measurable power incident on the cavity when matched and $v_{2}-v_{1}$ the width of the critically coupled cavity "dip " at the 3-dB points (half-power) in the klystron power mode [40]. A steel ball of $1.3 \mathrm{~mm}$ radius was placed exactly in the cavity center in a standard ESR tube. The microwave frequency was measured with a Marconi 2440 counter and the microwave power with a Marconi 6960 power meter in conjunction with the 6910 head. $v_{1}$ and $v_{2}$ were measured according to the reference [40].

We also carried out a measurement of $H_{1}$ by using a method based on the saturation broadening observed in a solution of PADS obeying the relationship [43]

$$
\Delta H_{\mathrm{pp}}^{2}=(4 / 3) \gamma^{-2} T_{2}^{-2}+(4 / 3) H_{1}^{2} T_{1} / T_{2}
$$

where $\Delta H_{\mathrm{pp}}$ is the derivative peak-to-peak linewidth, $T_{1}$ and $T_{2}$ the longitudinal and transverse electronspin relaxation time, respectively, of PADS, $\gamma$ the gyromagnetic ratio of the electron. Since for an aqueous solution of PADS at room temperature $T_{1} \cong T_{2}$ [43], $H_{1}$ can be obtained as a function of the microwave power $P_{\mathrm{i}}$ from an experimental plot of $\Delta H_{\mathrm{pp}}^{2}$ against $P_{\mathrm{i}}$. Another steady-state (cw) saturation study can be performed on PADS, whose saturation properties are well known [43]. The PADS methods is however more suitable for aqueous samples that have the same dimensions and dielectric and macroscopic properties as the PADS reference solution.

An alternative method for measuring $H_{1}$ values is to use point samples that are located within the sample of interest [44-46]. The best position is the centre of cavity where $H_{1}$ is maximal [41]. On type of point sample consists of a tiny crystal of NMP-TCNQ. Vistnes and Dalton [44] have presented a couple methods for $H_{1}$ determination using this crystal : for $H_{1} \lesssim 0.1 \mathrm{G}$ the method is based on a magnetization hysteresis parameter obtained from a magnetization hysteresis $(\mathrm{MH})$ spectrum, for $H_{1} \gtrsim 0.1 \mathrm{G}$ on linewidth measurements. As for the PADS method discussed above, the square of the peak-to-peak linewidth in the first harmonic spectrum from NMPTCNQ increases linearly with $H_{1}^{2}$. Since in this case $T_{1}$ and $T_{2}$ are not precisely known at present and the microwaves might be attenuated inside the crystal because of a small skin depth [44], an empirical calibration curve was constructed (Fig. 6 of Ref. [44]). The NMP-TCNQ crystal can be used for every type of sample (it is not water soluble) over a wide range of temperatures. Consequently, it has several advantages compared to PADS. Moreover, both NMPTCNQ methods are far more rapid than the perturbing sphere method. Our NMP-TCNQ crystal, fastened to a strip of Mylar film, was introduced into a glass microcapillary and placed in a standard ESR quartz tube exactly in the cavity centre. The ESR tube was, in turn, introduced into the temperature control quartz dewar and rotated by means of a goniometer in order to minimize the asymmetry of the lineshape arising from conductivity effects in the needle axis direction [44]. Another point sample useful for an accurate $H_{1}$ determination consists of a small drop of DPPH embedded in epoxy resin [46]. The value of $H_{1}$ can be determined by simply measuring the ESR signal height. In the presence of solvent in the sample, the signal intensity of the DPPH probe reflects both the dielectric loss and the lens effects, which are expressed by the cavity $Q$ and the filling factor $\eta$, respectively [40]. Below saturation, $\left(H_{1} \approx 0.2 \mathrm{G}\right)$ [46], the following equations [40] describe the effect of $Q$ and $\eta$ on the signal height $S$

$$
\begin{aligned}
& S \propto Q \eta P_{\mathrm{i}}^{1 / 2} \\
& S \propto(Q \eta)^{1 / 2} H_{1} .
\end{aligned}
$$

By knowing the absolute value of $H_{1}$ in the cavity in an 
independent experiment, the $H_{1}$ value for any other experimental setup is obtainable from the signal height $S$. For values of the magnetic microwave field at the sample higher than $0.2 \mathrm{G}$, the method already discussed should be carried out at power levels well below saturation, giving rise to the proportionality

$$
H_{1}=\alpha P_{i}^{1 / 2} \text {. }
$$

The incident power, belonging to the desired value of $H_{1}$ above saturation, is then found from equation (6). DPPH measurements have been performed in the absence and in the presence of the lipid sample. In the first case the sample holder for the DPPH drop, attached to a tiny glass fiber, was the same as for the TCNQ crystal capillary. In the second case the DPPH was attached directly to the lipid sample capillary at the same position as the lipid point sample.

The other instrumental parameter we considered for calibration, was the modulation amplitude $H_{\mathrm{m}}$. The value of $H_{\mathrm{m}}$ in the centre of the cavity was determined by the NMP-TCNQ crystal which is characterized by a very small natural linewidth of $\cong 0.13 \mathrm{G}$. The conventional first harmonic absorption ESR spectrum was recorded with a modulation frequency of $25 \mathrm{kHz}$ and a high modulation amplitude $(5 \mathrm{G})$, the peak-topeak distance in the over-modulation spectrum giving the exact value of $H_{\mathrm{m}}$. Different from $H_{1}$, the calibration of $H_{\mathrm{m}}$ at the cavity center is insensitive to the cavity filling. Once calibrated for a particular cavity, $H_{\mathrm{m}}$ is known for all future experiments.

Data presented below, which proved to be quite reproducible, are based on repeated experiments.

\section{Results and discussion.}

As already mentioned, linear ESR experiments involve the application of a microwave radiation field so weak that the induced spin transitions fail to dominate electron spin-lattice processes, the population of the spin levels remaining unperturbed from their Boltzmann equilibrium values. In this operation mode the only effect of time dependent events modulating magnetic interactions is to influence the phase coherence of the precessing spins (spin-spin relaxation processes), with a resultant effect upon resonance linewidth. But linear ESR is not able to follow very slow nitroxide motions, this motional region being characterized by the relationship $\Delta \omega \tau_{\mathrm{c}} \gg 1$ [25], where $\Delta \omega$ is the anisotropy of the spin label magnetic interaction. In fact, the above inequality leads to an ordinary ESR spectrum which is for nonoriented, randomly distributed, molecules in the sample the same as that for a rigid powder. The inability to distinguish any dynamic feature in such cases, led to the development of non-linear ESR techniques sensitive to the spin label motion in this time domain. The non linearity stems from the fact that in these spectroscopic approaches the applied microwave radiation field is so intense as to overcome the spin-lattice rela- xation effects, thereby perturbing the spin levels from their Boltzmann population equilibrium values. In the presence of microwave saturation, the rotational diffusion which the spin labelled molecules undergo modulates the anisotropic interactions and, since this very slow rotational diffusion rate is comparable to the spin-lattice relaxation time $\left(0.01 T_{1} \approx \tau_{c} \approx 100 T_{1}\right)$ [25], transfer of saturation occurs. This gives rise to spectral diffusion which manifests itself differently in different parts of the spectrum.

The important physical fact is that under these conditions of saturation, the system is sensitive to the rate at which $H$, the total magnetic field felt by the spins, goes through its resonant value. On the other hand, this rate depends on the fluctuations of the local magnetic field sensed by the electrons, $H_{1}$, due to the diffusion of the spin label; $H_{1}$ is modulated by molecular motions of correlation time $\tau_{\mathfrak{c}}$, which thus sweep $H$ past its resonance value and influence the signal $\left(\frac{\mathrm{d} H_{1}}{\mathrm{~d} t} \propto \frac{1}{\tau_{\mathrm{c}}} H_{1}\right)$. Moreover, this rate depends on the frequency of the modulation field $\left(\frac{\mathrm{d} H}{\mathrm{~d} t} \propto \omega_{\mathrm{m}} H_{\mathrm{m}}\right)$, which now has a double function : the detection of the signal, as in linear ESR experiments, and the creation of a proper rate of passage of $H$ through resonance, which, in many cases, effects in the so-called " adiabatic rapid passage $"$ situation [47] :

$$
\frac{H_{1}}{T_{1}} \ll\left|\frac{\mathrm{d} H}{\mathrm{~d} t}\right| \ll \gamma H_{1}^{2} .
$$

Under these conditions the sweep of the Zeeman magnetic field through resonance is so slow that, in the rotating frame, the resultant magnetization vector lies along the effective field throughout the sweep, but is still fast compared to $T_{1}$. In order to gather the maximum information on the saturation behaviour it is convenient to have $T_{1}=\omega_{\mathrm{m}}^{-1}$ [22]. Since $T_{1}$ is of the order $10^{-6} \mathrm{~s}$ for nitroxide spin labels [22], the passage is no longer adiabatic if $\tau_{\mathrm{c}}=5 \times 10^{-5} \mathrm{~s}$ [25]; in this case the magnetization vectors are no longer aligned along the effective fields, and the experimental observable is altered. This situation creates a phase lag which therefore contains information on the molecular tumbling in the motional range $10^{-7} \approx$ $\tau_{\mathrm{c}} \approx 10^{-3} \mathrm{~s}$ [25]. Conventional ESR spectroscopy works at low microwave power and the absorption signal can be written as an expansion of the harmonics of the modulation frequency $\omega_{\mathrm{m}}$

$$
A\{H(t)\}=\sum_{n=0}^{\infty} a_{n} \cos n \omega_{\mathrm{m}} t .
$$

Moreover, phase-sensitive detection is used to observe the signal; when the reference frequency of the phasesensitive detector is set at $\omega_{\mathrm{r}}$, two signals can in general be detected, one which oscillates with $\cos \omega_{\mathrm{r}} t$ (the 
in-phase signal), and one which oscillates with $\sin \omega_{\mathrm{r}} t$ (the quadrature or out-of-phase signal). From equation (8), it is clear that the quadrature signals vanish for all the harmonics. On the other hand, we have already mentioned that under saturation conditions at high microwave power, and if $\omega_{\mathrm{m}}^{-1}=T_{1}=\tau_{\mathrm{c}}$, the spin system is not able to follow the field modulation, thus giving rise to a phase-shift $\Phi_{n}$ in the signal $A\{H(t)\}$

$$
A\{H(t)\}=\sum_{n=0}^{\infty} a_{n} \cos \left(n \omega_{\mathrm{m}} t+\Phi_{n}\right) .
$$

This expression can be separated into the in-phase and the out-of phase signal. In order to amplify slight modifications of the spectral line shape the quadrature signals, sensitive to very slow $\tau_{\mathrm{c}}$ values, are displayed in the second derivative of the absorption spectrum.

As already mentioned, the rate of spectral diffusion depends on the angle between the magnetic field and the $2 p \pi$ orbital of the nitroxide axis [1], and therefore on the resonance value of the magnetic field. This means that saturation transfer leaves some spectral points, the so-called " turning points ", totally unaffected, and has its maximum effect at field values in between them. ST-ESR spectra can therefore be well characterized by three independent line shape parameters [22], $L^{\prime \prime} / L, C^{\prime} / C$ and $H^{\prime \prime} / H$, shown in figure 4. Thomas et al. [22] have developed a method to obtain rotational correlation times from these spectral parameters. In fact, they have calibrated them against $\tau_{\mathrm{c}}$ by applying the Debye equation for Brownian rotational diffusion to spin-labelled macromolecules. This procedure is the cornerstone of the ST-ESR method since, up to now, almost all experimental spectra have been interpreted on the basis of those calibration curves.

However these spectra are very much influenced by the experimental settings [22], i.e. the value of the magnetic component of the microwave field, $H_{1}$, the modulation amplitude, $H_{\mathrm{m}}$, the setting of the phase $\Phi$ of the phase-sensitive detector, and the spectrometer must be adjusted with more care than in conventional ESR. We report first the results concerning these instrumental parameters which we determined in our apparatus.

As already mentioned, PADS is not very suitable for measuring the incident microwave magnetic field strength on our lipid sample. However, some PADS experiments were carried out in order to compare our results with some literature results [35-36], under the same instrumental and sample holder conditions. The proportionality constant between $H_{1}^{2}$ and $P_{\mathrm{i}}, k$, $\left(H_{1}^{2}=k P_{\mathrm{i}}\right)$, turned out to be in good agreement with those in the literature.

Figure 2 shows the plot of $H_{1}^{2}$ versus $P_{i}$, that has been obtained with the perturbing sphere method at room temperature, using equation (2). The slope of the line determined a value of $k=2.2 \mathrm{G}^{2} / \mathrm{W}$.

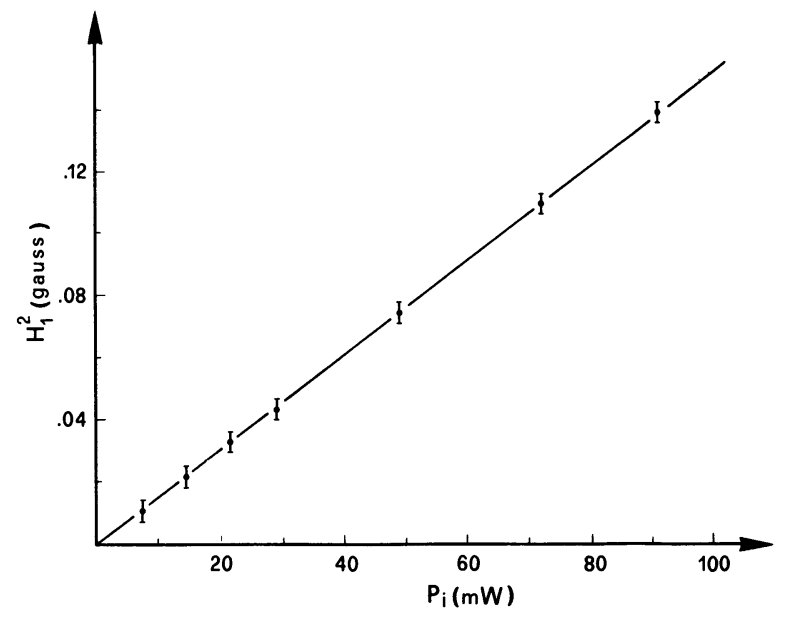

Fig. 2. - Microwave power dependence of the square of the microwave magnetic field incident on a steel ball $(\phi=$ $2.6 \mathrm{~mm}$ ) in the centre of the Varian $\mathrm{TE}_{102}$ rectangular cavity, at room temperature (perturbing sphere method). The sphere was introduced in a standard ESR quartz tube inserted in the temperature control quartz Dewar.

The square of the derivative peak-to-peak linewidth, $\Delta H_{\mathrm{pp}}^{2}$, for the NMP-TCNQ crystal as a function of $P_{\mathrm{i}}$ is given in figure 3. A comparison with the experimental curve $\Delta H_{\mathrm{pp}}^{2}\left(H_{1}^{2}\right)$ obtained by Vistes and Dalton [44] led to a $k$ value of $2.65 \mathrm{G}^{2} / \mathrm{W}$. A possible interpretation of this discrepancy may be given as follows : the steel ball volume is greater than the crystal one and broadening behaviour may arise because of the field inhomogeneities within the cavity.

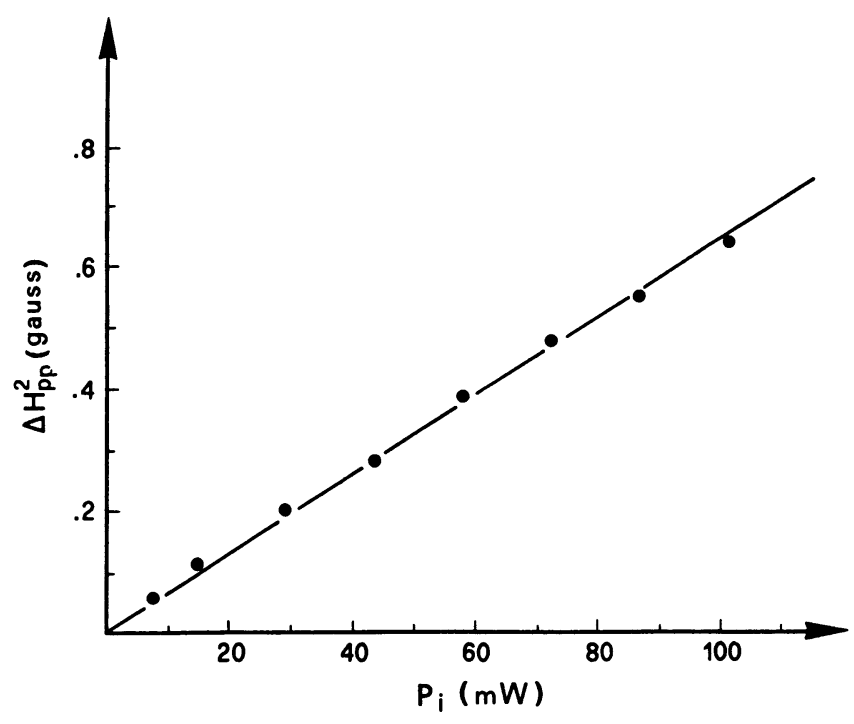

Fig. 3. - Microwave power dependence of the square of the derivative peak-to-peak linewidth of a NMP-TCNQ crystal positioned in the Varian $\mathrm{TE}_{102}$ cavity centre, at room temperature. Sample holder as in figure 2. Modulation amplitude $=0.05 \mathrm{G}$. Experimental error does not exceed circle diameter. 
We also performed TCNQ experiments at different temperature values; they resulted in a very slight variation of $k$ with temperature, namely $4.5 \times$ $10^{-3} \mathrm{G}^{2} / \mathrm{W}^{\circ} \mathrm{C}$ (data not shown).

The above experiments were undertaken in the absence of the bipolar lipid sample. By using the DPPH method we found that the introduction of the GDNT point sample increases the microwave magnetic field in the centre of the cavity by $4 \%$. Therefore, for reason of convenience, so as to determine the $H_{1}$ value on the lipid sample, we first performed experiments without the sample and then took into account the above mentioned increase. We believe that in our case the results obtained with the TCNQ crystal are more reliable than those obtained through the method of the perturbing sphere. In fact, the lipid sample size is more similar to that of the crystal than to that of the ball. Therefore, the $k$ value chosen for our lipid measurement is $k=2.86 \mathrm{G}^{2} / \mathrm{W}$ at a temperature of $20^{\circ} \mathrm{C}$. Furthermore, we compensated for the temperature effects by changing the incident power accordingly before each spectrum was recorded at a different temperature.

Figure 4 shows conventional first harmonic, inphase, ESR spectra at some temperature values, and the corresponding second harmonic, $90^{\circ}$ out-ofphase, absorption ESR spectra. As can be seen, the conventional signals show the typical pattern of rigidlimit powder line shapes and this pattern does not change noticeably with increasing temperature, at least up to $50^{\circ} \mathrm{C}$. In particular, only slight variations in the $2 A_{z z}$ splitting (this parameter is commonly used to describe empirically the fluidity of the lipid system when only conventional ESR experiments are performed [48]) are observed with increasing temperature. This behaviour suggests that the system undergoes molecular motions satisfying the above mentioned relationship $\Delta \omega \tau_{c} \gg 1$ and in this motional range conventional ESR is not sensitive to changes occurring in the dynamics of the system. By contrast the ST-ESR spectra are very much dependent on the temperature and the spectral parameters are noticeably different from one spectrum to another. These ST-ESR spectra were recorded at $H_{1}=0.25 \mathrm{G}$ on the sample and $H_{\mathrm{m}}=5 \mathrm{G}$. From the $k$ values obtained from the TCNQ experiments, the first condition required a microwave power of $22 \mathrm{~mW}$ at $20^{\circ} \mathrm{C}$, decreasing slightly with increasing temperature up to a value of $20 \mathrm{~mW}$ at $80^{\circ} \mathrm{C}$. As regards the second condition, the TCNQ measurements have shown a reliability of the modulation amplitude setting device of about $1 \%$.

The values of $C^{\prime} / C$ and $L^{\prime \prime} / L\left(H^{\prime \prime} / H\right.$ is not measurable, as already seen by other authors [32]), at some temperatures, and the respective correlation times are given in table I. As can be seen, molecular motions belong to the very slow motional region, and the correlation time determined from $C^{\prime} / C$ shows a value of $1.3 \times 10^{-7} \mathrm{~s}$ even at $80^{\circ} \mathrm{C}$. Correlation time values found from the $L^{\prime \prime} / L$ calibration curve are quite different from those obtained with the $C^{\prime} / C$ value. For instance, at room temperature the first spectral parameter leads to $\tau_{\mathrm{c}}=10^{-5} \mathrm{~s}$, while the second leads to $\tau_{c}=3 \times 10^{-6} \mathrm{~s}$. This behaviour suggests the presence of anisotropic motion [31], at least up to $70^{\circ} \mathrm{C}$ (at higher temperatures $L^{\prime \prime}$ is no longer mea-

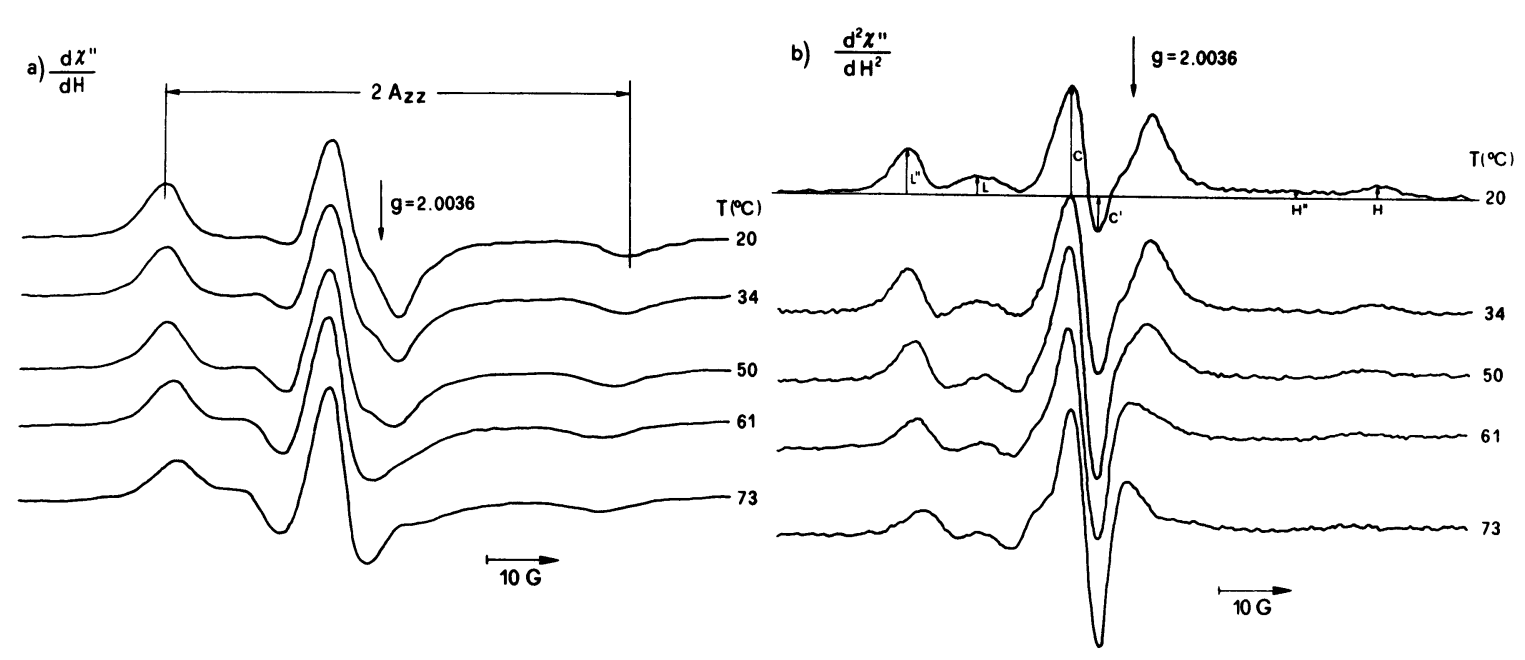

Fig. 4. - a) Conventional first harmonic, in-phase, absorption ESR spectra at some temperature values of 5SASL-labelled dry GDNT point samples. The samples are randomly oriented and the label/lipid molar ratio is about $1 \%$. They are introduced into a standard ESR quartz tube inserted, in turn, in the temperature control quartz dewar, and then positioned exactly in the center of the cavity. ESR settings as follows : magnetic field sweep rate $100 \mathrm{G}$ in $4 \mathrm{~min}$, time constant $0.25 \mathrm{~s}$, modulation amplitude $1.25 \mathrm{G}$ and microwave power level $6 \mathrm{~mW}$.

b) ST-ESR spectra at some temperature values in the second harmonic, $90^{\circ}$ out-of-phase, absorption mode of 5SASLlabelled dry GDNT point samples. Sample conditions as in a). ESR settings as follows : magnetic field sweep rate $100 \mathrm{G}$ in $8 \mathrm{~min}$, time constant $0.128 \mathrm{~s}$, modulation amplitude $5 \mathrm{G}$ and microwave power level varying linearly from $22 \mathrm{~mW}$ at room temperature to $20 \mathrm{~mW}$ at $80^{\circ} \mathrm{C}$. 
Table I. - $C^{\prime} / C$ and $L^{\prime \prime} / L$ ratios from ST-ESR of SSASL - labelled GDNT, and the related correlation times, in the temperature range $20-80^{\circ} \mathrm{C}$. Correlation times were calculated through the calibration curves of Thomas et al. [3].

\begin{tabular}{|c|c|c|c|c|}
\hline$T\left({ }^{\circ} \mathrm{C}\right)$ & $C^{\prime} / C$ & $\tau_{\mathrm{c}}\left(C^{\prime} / C\right)$ & $L^{\prime \prime} / L$ & $\tau_{\mathrm{c}}\left(L^{\prime \prime} / L\right)$ \\
\hline 20 & -0.28 & $3.0 \times 10^{-6}$ & 0.40 & $1.0 \times 10^{-5}$ \\
30 & -0.39 & $2.0 \times 10^{-6}$ & 0.30 & $6.0 \times 10^{-6}$ \\
40 & -0.55 & $1.0 \times 10^{-6}$ & 0.25 & $3.5 \times 10^{-6}$ \\
55 & -0.73 & $3.0 \times 10^{-7}$ & 0.17 & $1.5 \times 10^{-6}$ \\
70 & -0.90 & $1.8 \times 10^{-7}$ & 0.10 & $5.0 \times 10^{-7}$ \\
80 & -1.0 & $1.3 \times 10^{-7}$ & - & - \\
\hline
\end{tabular}

surable). Confirmation of this hypothesis comes from plotting the $L^{\prime \prime} / L$ ratio $v s$. $C^{\prime} / C$, for increasing temperatures, as indicated by Johnson et al. [33]. A possible interpretation is that $C^{\prime} / C$ yields information on the motion around the long molecular axis of the spin label, whereas $L^{\prime \prime} / L$ gives the correlation time for motion of the long axis itself [31]. The plots of these two spectral parameters $v s$. temperature are given in figure 5 . A plateau in the temperature range $45 \div 50^{\circ} \mathrm{C}$ suggesting that the system undergoes a broad structural transition at this temperature. X-ray crystallografic studies also performed on the same biological system [39] indicated a transition in the same temperature range from rectangular, $P_{\alpha}$, to a hexagonal, $H_{\text {III }}$, phase.

The most remarkable feature arising from this ST-ESR study lies on the high values of the correlation times. Since 5SASL spin probes yield information about the lipid portion near the nonitol polar head [38] (strictly speaking we measure the mobility of the spin probe, but this motion is assumed to be conforming with the molecular motion of lipid molecules in which the spin probe is dissolved in), these results indicate the ability of the nonitol polar heads to establish highly cooperative interactions even at high temperatures $\left(80^{\circ} \mathrm{C}\right)$. Since the nonitol polar head is exposed towards the outside of the cell [38], such strong interactions might explain the great stability of the plasma membrane even under those extreme environmental conditions corresponding to the physiological ones.

\section{Conclusions.}

An accurate determination of the magnetic instrumental parameters upon which the ST-ESR line shapes are critically dependent, allowed us to perform a non-linear ESR study in order to gain dynamic
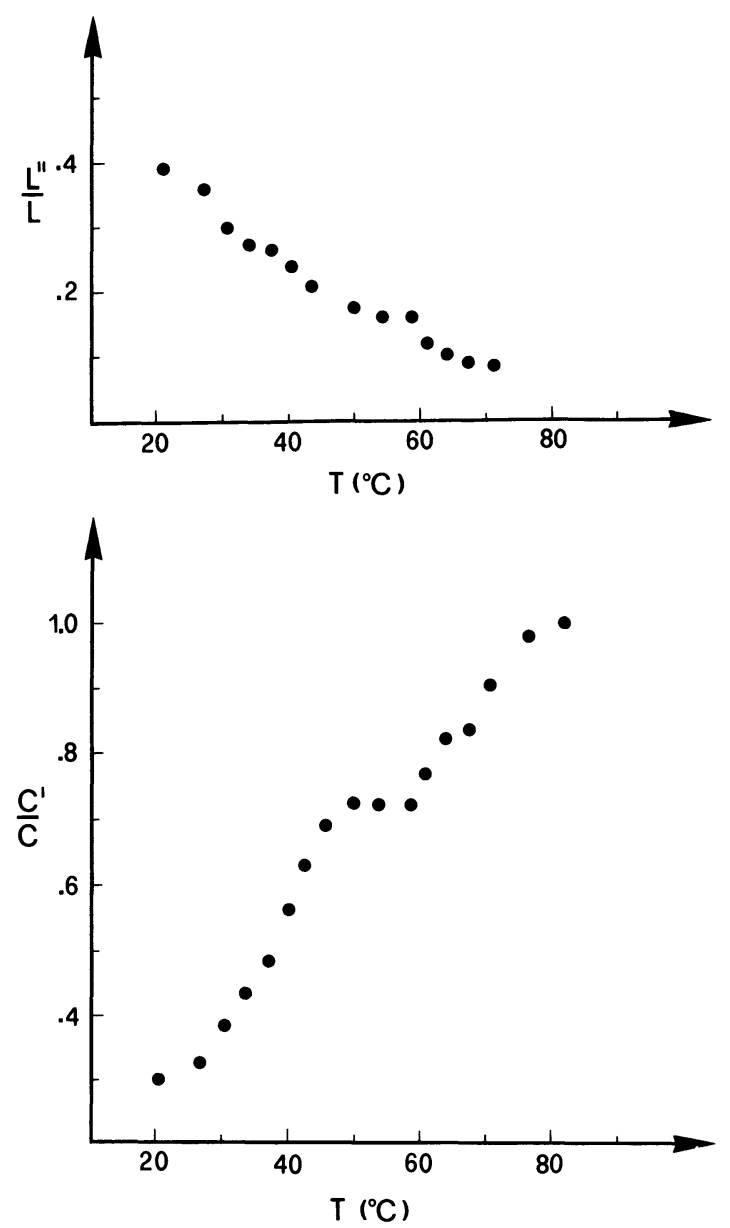

Fig. 5. - Temperature dependence of the spectral ratios $L^{\prime \prime} / L$ (a) and $C^{\prime} / C$ (b) from ST-ESR spectra of GDNT labelled with 5SASL. Sample conditions and ESR settings as in figure $4 \mathrm{~b}$. Experimental error does not exceed circle diameter.

information on GDNT lipids extracted from an extreme thermoacidophilic archaebacterium. The correlation times turned out to be unusually large, as compared with the ordinary lipid ones, and the molecular motion showed an anisotropic nature, even at very high temperatures.

Even if lipid-protein interactions cannot be ruled out, this behaviour may be partly responsible for the great stability of the plasma membrane which allows these bacteria to withstand the high physiological temperature and the high $\mathrm{pH}$ gradient.

\section{Acknowledgments.}

This work has been partly supported by CNR and MPI grants. Thanks are due to Dr. A. I. Vistnes for the kind gift of NMP-TCNQ crystals and to Mrs. R. Mencarini for typing the manuscript. 
References

[1] Hemminga, M. A., Chem. Phys. Lipids, 32 (1983) 323 and references therein.

[2] Redfield, A. G., Adv. Magn. Res. 1 (1966) 1.

[3] Schindler, H. G. and Seelig, J., J. Chem. Phys., 59 (1973) 1841.

[4] Abragam, A., Principles of Nuclear Magnetism (Claredon Press, Oxford) 1960.

[5] Glarum, S. H. and Marshall, J. A., J. Chem. Phys. 46 (1967) 55.

[6] Nordio, P. L., Rigatti, G. and Segre, U., J. Chem. Phys. 56 (1972) 2117.

[7] Hemminga, M. A., Chem. Phys. Lip. 14 (1975) 141 and 151.

[8] Cannistraro, S. and Indovina, P. L., Nuovo Cimento, 4D (1984) 206.

[9] McCalley, R. C., Shimshick, E. J. and McConnell, H. M., Chem. Phys. Lett. 13 (1972) 115.

[10] Freed, J. H., in : L. J. Berliner (Ed.) Spin Labelling Theory and Applications, Vol. 1 (Academic Press, New York, N.Y.) 1976, p . 53-132.

[11] Freed, J. H., Bruno, G. V. and Polnaszek, C. F., J. Phys. Chem. 75 (1971) 3385.

[12] Jansen, S. J. K., in : L. T. Muus and P. W. Atkins (Eds.) Electron Spin Relaxation in Liquids (Plenum Press, New York) 1972, p. 71-88.

[13] Gordon, R. G. and Messenger, T., in : L. T. Muus and P. W. Atkins (Eds.) Electron Spin Relaxation in Liquids (Plenum Press, New York) 1972, p. 341381.

[14] Polnaszek, C. F., Bruno, G. V. and Freed, J. H., J. Chem. Phys. 58 (1973) 3185.

[15] Polnaszek, C. F. and Freed, J. H., J. Phys. Chem. 79 (1975) 2283.

[16] Meirovitch, E. and Freed, J. H., J. Phys. Chem. 84 (1980) 3281.

[17] Polnaszek, C. F., Marsh, D. and Smith, I. C. P., J. Magn. Reson. 43 (1981) 54.

[18] Goldman, S. A., Bruno, G. V. and Freed, J. H., J. Phys. Chem. 76 (1972) 1858.

[19] Goldman, S. A., Bruno, G. V. and Freed, J. H., J. Chem. Phys. 59 (1973) 3071.

[20] Hyde, J. S., Smigel, M. D., Dalton, L. R. and DalTON, L. A., J. Chem. Phys. 62 (1975) 1655.

[21] Hyde, J. S., Phys. Rev. 119 (1960) 1483.

[22] Thomas, D. D., Dalton, L. R. and Hyde, J. S., J. Chem. Phys. 65 (1976) 3006.

[23] Thomas, D. D. and McConnell, H. M., Chem. Phys. Lett. 25 (1974) 470.

[24] Dalton, L. R., Robinson, B. H., Dalton, L. A. and Coffey, P., Adv. Magn. Reson. 8 (1976) 149.

[25] Hyde, J. S. and Dalton, L. R., in : L. J. Berliner
(Ed.) Spin Labelling Theory and Applications, Vol. 2 (Academic Press, New York, N.Y.) 1979, p. 1-70.

[26] Robinson, B. H. and Dalton, L. R., J. Chem. Phys. $72(1980) 1312$.

[27] Perkins, R. C., Lionel, T., Robinson, B. H., Dalton, L. A. and Dalton, L. R., Chem. Phys. 16 (1976) 393.

[28] Mailer, C. and Miller, D. M., J. Magn. Reson. 32 (1978) 289.

[29] Galloway, N. B. and Dalton, L. R., Chem. Phys. 41 (1979) 61.

[30] Robinson, B. H. and, Dalton, L. R., Chem. Phys. 54 (1981) 253.

[31] MARSH, D., Biochemistry 19 (1980) 1632.

[32] Delmelle, M., Butler, K. W. and Smith, I. C. P., Biochemistry 19 (1980) 698.

[33] Johnson, M. E., Lee, L. and Fung, L. W. M., Biochemistry 21 (1982) 4459.

[34] Beth, A. H., Wilder, R., Wilkerson, L. S., Perkins, R. C., Meriwether, B. P., Dalton, L. R., Park, C. R. and PARK, J. H., J. Chem. Phys. 71 (1979) 2074.

[35] Fajer, P. and Marsh, D., J. Magn. Reson. 49 (1982) 212.

[36] Delmelle, M., J. Magn. Reson. 51 (1983) 245.

[37] Gliozzi, A., Paoli, G., De Rosa, M. and GambaCORTA, A., Biochim. Biophys. Acta 735 (1983) 234.

[38] Bruno, S., Cannistraro, S., Gliozzi, A., de Rosa, M. and Gambacorta, A., Eur. Biophys. J. 13 (1985) 67.

[39] Gulik, A., Luzzati, V., De Rosa, M. and GambaCORTA, A., J. Mol. Biol. in press.

[40] Dalal, D. P., Eaton, S. S. and Eaton, G. R., J. Magn. Reson. 44 (1981) 415.

[41] Poole, C. P. Jr., Electron Spin Resonance (Wiley, Interscience, New York) 1976.

[42] Gintzon, E. L., Microwave Measurements (McGrawHill Book Co., New York) 1957.

[43] Kooser, R. G., Volland, M. V. and Freed, J. H., J. Chem. Phys. 50 (1969) 5243.

[44] Vistnes, A. I. and Dalton, L. R., J. Magn. Reson. 54 (1983) 78.

[45] Hemminga, M. A., Reinders, J. H. and de Jager, P. A., J. Magn. Reson. 58 (1984) 428.

[46] Hemminga, M. A., Leermakers, F. A. M. and DE JAGER, P. A., J. Magn. Reson. 59 (1984) 137.

[47] PAKe, G. E., Paramagnetic Resonance (Benjamin, New York) 1962.

[48] Gaffney, B. J. and MCNamee, C. M., Methods in Enzymology 32 (1974) 161. 